\title{
Arteries of the chorion of pregnant women with arterial hypertension
}

\author{
Gansburgskiy $\mathrm{AN}^{1 *}$ and Yaltsev $\mathrm{AV}^{2}$ \\ ${ }^{1}$ Department of histology, cytology and embryology, Yaroslavl State Medical University, Yaroslavl, Russia \\ ${ }^{2}$ Pathological anatomy with the course of clinical pathology, Yaroslavl State Medical University, Yaroslavl, Russia
}

\begin{abstract}
The 31 last women 28-35 years were studied at a gestation period of 35-37 weeks with arterial pressure of 140/110-180/120 mm. Hg. The comparison group included 10 successions of women of the same age with a pressure of 110/80-120/80 mm. Hg. in the physiological course of pregnancy. The mass of the afterbirths in both cases was 450-650 g. Histological, histochemical, immunohistochemical and morphometric methods were used to study the arteries of the chorionic plate of the placenta. Clinical studies included dopplerometry and pulsed doppler echocardiography on the Combison-530 Kretztechnik (a sensor with a frequency of $3.5 \mathrm{MHz}$, a filter frequency of $100 \mathrm{~Hz}$ ). The quantitative data were processed by the method of variational statistics. Conducted clinical functional studies allowed to establish in pregnant women with hypertension an increase in the pulsation index of the umbilical cord and at the same time a decrease in the minute volume of blood (the cardiac output of the mother). Registered changes in blood flow indicate the development of fetal hypoxia. In conditions of increased pressure, hypertrophy of the wall of the chorion arteries develops, the number of arteries with a functional layer of smooth myocytes in the middle shell increases, as well as vessels of the closing type with intimal muscles actively participating in the regulation of the organ circulation. $\mathrm{n}$ arteries with a hypertrophic wall, a high proliferation index of Ki- 67 major cell populations were revealed. The level of proliferation of smooth myocytes in the arteries of the chorionic plate in the case of hypertension exceeds by a factor of 1.5 the values of the index of the comparison group. The significant development of smooth myocytes in tunica internal leads to a narrowing of the arteries' lumen, in which only a sliced space is left for the flow of blood, and in some cases-to complete obliteration of the lumen. In conditions of arterial hypertension in the vascular wall, dystrophic changes of smooth myocytes, stromal edema and intravascular thrombi are detected. Identified structural changes lead to a significant decrease in blood volume, the development of acute or chronic placental anemia and, therefore, placental insufficiency and hypoxic conditions in the fetus.
\end{abstract}

Abbreviations: AH: Arterial hypertension; AP: Blood pressure; SM: Smooth myocytes; 1/min: Liter per minute; HP: Chorionic plate; YSMU: Yaroslavl state medical university

\section{Introduction}

The study of the vascular bed of the placenta of pregnant women with arterial hypertension $(\mathrm{AH})$ has important practical and theoretical significance [1-3]. Structural changes in the arterial vessels of a child's place can affect placental hemodynamics and become a decisive factor in fetal formation [4-7]. The most important in this case are the arteries of the chorionic plate (HP), which are arteries of large caliber and thereby providing the main blood flow. In the morphological laboratories of the YSMU, variants of compensatory-adaptive changes in the vessels of $\mathrm{HP}$ in cases of chronic placental insufficiency are demonstrated $[8,9]$. In the literature, information on the morphofunctional features of the arterial vessels of the HP of the placenta in conditions of hypertensive syndrome in pregnant women is few and scattered [10,11].

The aim of this work is to evaluate the morphofunctional features of the chorionic plate arteries of the placenta of pregnant women with arterial hypertension and to analyze their effect on the placental-fetal circulation.

\section{Material and methods}

The 31 last women 28-35 years were studied at a gestational age of $35-37$ weeks with blood pressure from $140 / 110$ to $180 / 120 \mathrm{~mm}$. Hg. The comparison group included 10 successions of women of the same age with a blood pressure of 110/80-120/80 mm. Hg. in the physiological course of pregnancy. The mass of the afterbirths in both cases was 450-650 g. The material was obtained from the maternity hospitals of Yaroslavl, the pathomorphological examination was performed in the Clinical Hospital of the name of Yaroslavl N.V. Solovyov. The work was approved by the Ethics Committee of the YSMU (Minutes No. 19 of December 5, 2017). From the fetal membrane of the placenta excised fragments in the central, middle and marginal part measuring $7 \times 7 \mathrm{~mm}$ and fixed in $10 \%$ neutral formalin and Carnoy fluid. Serial sections 4-6 $\mu \mathrm{m}$ thick were stained with hematoxylin with eosin, Masson, van Gieson, the glycogen content was detected by the Schick reaction (control with amylase). Immunohistochemical study was performed on dewaxed sections with an indirect immunoperoxidase method using the Ki-67 proliferation marker (Ventana, USA), followed by staining with Mayer's hematoxylin. The reaction was performed on the immunohistostereer Roche Benchmark XT Ventana (USA). The number of immunopositive nuclei and the proliferation index were counted. In this case, 1000 nuclei in the endothelium and smooth myocytes (SM) of the arteries were taken into account. The external and internal diameters of arteries were measured with a screw ocular micrometer MOV-1-15x (LOMO, Russia), the wall thickness and the cross-sectional area of the middle shell were calculated [12].

Correspondence to: Gansburgskiy Andrey Nikolaevich, Doctor of Medical Sciences Professor, Russia, E-mail: profang@mail.ru

Key words: arterial hypertension, chorionic artery arteries, smooth myocytes

Received: February 07, 2018; Accepted: February 21, 2018; Published: February 27,2018 
Clinical studies included dopplerometry, which determined the pulsation index of the umbilical cord, and pulsed doppler echocardiography on the Combison-530 Kretztechnik (a sensor with a frequency of $3.5 \mathrm{MHz}$, a filter frequency of $100 \mathrm{~Hz}$ ), which allows one to estimate the minute volume of blood (cardiac output the mother). The quantitative data were processed by the method of variational statistics. The significance of the differences was judged by the value of the t-test of the Student.

\section{Results}

Clinical observations showed that in healthy women of the comparison group the pulsation index of the umbilical cord is $0.86 \pm 0.07$, and in $\mathrm{AH}$ conditions the index increases to $1.32 \pm 0.09$. The minute volume of blood in women with a physiological course of pregnancy is equal to $5,837 \pm 1,095 \mathrm{l} / \mathrm{min}$, and with hypertension it is reduced to $4,814 \pm 1,778 \mathrm{l} / \mathrm{min}$.

Morphometric examination has established that the external diameter of the arteries of $\mathrm{HP}$ in women with $\mathrm{AH}$ increases insignificantly (Table 1). In this group, the lumen of the arteries decreases almost 3 times $(2.8 \pm 0.2 \mu \mathrm{m}, \mathrm{p}<0.01)$. The latter indicates a decrease in the capacity of arterial vessels of HP. In women with AH, attention is drawn to a significant (2-fold) increase in the thickness of the vascular wall. The cross-sectional area of the middle shell increases by $164.8 \%(\mathrm{p}<0.01)$. The findings indicate that the thickening of the artery walls is due to the hypertrophy of the tunica media and appears to be a response to $\mathrm{AH}$ in the arterial basin. Not infrequently this leads to an almost complete narrowing of the lumen of the arteries (Figure 1A).

A significant part of the arteries of the HP of the placenta in conditions of a normally occurring pregnancy, as well as with $\mathrm{AH}$, have some structural features. The middle shell is mainly represented by bundles of obliquely longitudinally oriented SM, which is proposed to be called "functional" $[2,13]$. An important feature of the arteries is the developed reticulin and elastic tunica media and the presence of interlacing bundles of collagen fibers around the outer shell (Figure 1B). It should be noted that in placentas without pathology of pregnancy the number of such vessels in HP is almost $40 \%(38.6 \pm 1.4 \%)$. While in placentas with $\mathrm{AH}$ their share reaches $60 \%(59.2 \pm 3.1 \%)$, that is, it increases almost 1.5 times. The cytoplasm of the SM middle shell is characterized by a high content of glycogen (Figure 1C). In arteries with hypertrophic wall, a high index of proliferation of Ki-67 major cell populations were revealed (Figure 1D, Table 2). The index of Ki67 immunopositive nuclei in the blood vessels of HP in pregnancy with $\mathrm{AH}$ was higher. Thus, the level of proliferation of SM in the arteries of $\mathrm{HP}$ in the case of hypertension exceeds by a factor of 1.5 the values of the index of the comparison group.
In the arteries of HP in women with $\mathrm{AH} 2.7$ times more often detected vessels with powerful bundles of GM in the inner shell (Figure 2A). Often this feature of tunica interna leads to a pronounced narrowing of the lumen of the arteries, in which only a sliced space is left for the flow of blood, and in some cases causes complete obliteration of the lumen. In the arteries with a functional layer in the wall, dystrophic changes of smooth myocytes (Figure 2B), edema of the stroma (Figure 2C) and intravascular thrombi (Figure 2D) are found.

\section{Discussion}

Clinical functional studies involving dopplerometry and pulsed doppler echocardiography have made it possible to increase the pulsation index of the umbilical cord in pregnant women with $\mathrm{AH}$ and simultaneously decrease the minute volume of the blood (the cardiac output of the mother). Registered changes in blood flow indicate the development of fetal hypoxia $[1,3]$.

Morphological methods in HP of the placenta revealed the development of a complex of structural changes in the arteries of compensatory-adaptive and pathological character. In conditions of increased pressure, the hypertrophy of the arterial wall of HP develops, the number of arteries with a functional layer of smooth myocytes in the middle shell increases, as well as vessels of the closing type with intimal muscles actively participating in the regulation of the organ circulation. These morphological transformations, in our opinion, and the literature data have a compensatory-adaptive character $[8,14,15]$. It should be noted the significant contractile potential of the arteries of $\mathrm{HP}$, which is provided by a high content of glycogen, which is related to the formation of energy of muscle contraction [16]. The histological structure of the arteries with the functional layer of the SM in the middle layer of HP is in many respects the same as the morphology of the umbilical arteries. Their smooth muscle elements are represented by clearly delineated longitudinal and circular layers, capable of actively contracting and, probably, have peristaltic activity [2]. Arteries with a skew-layered layer of SM in the intima are the structural element of a self-regulating circulatory system between the uterus and placenta with low resistance to blood flow and not responding to conventional neurogenic stimuli [14].

The most important property of the fetal circulation in the placenta, which by the 39-40 week completes its development [14], is a slowing of blood flow and a drop-in pressure in the arterial system. This is facilitated by high branching, anastomosing, rotations at right angles, significantly extending the blood flow in the arteries of HP and [14,17]. Unlike straight segments with a laminar nature of motion, the regions of bends and flow divisors are special areas with turbulence, vorticity, recirculation and a complex character of the shear stress distribution

Table 1. Morphometric characteristics of the arteries of the chorionic plate of pregnant women $(\mathrm{M} \pm \mathrm{m})$

\begin{tabular}{|c|c|c|c|}
\hline Group of observations & Outside diameter, mkm & Inside diameter, micron & $\begin{array}{c}\text { Thickness, mkm } \\
\text { Normal flow of pregnancy }\end{array}$ \\
\hline Arterial hypertension & $90,0 \pm 1,6$ & $52,2 \pm 0,8$ & $19,6 \pm 0,8$ \\
\hline section shell, $\boldsymbol{\mu m m}^{\mathbf{2}}$ & $4236 \pm 89$ \\
\hline
\end{tabular}

Note: Differences are significant compared to the control

"for $\mathrm{P}<0.01$

Table 2. Index of Ki-67 immuno-positive nuclei of cellular populations of arteries of chorionic plate of pregnant women (M $\pm \mathrm{m})$

\begin{tabular}{|c|c|c|}
\hline Group of observations & Endothelium & Smooth myocytes \\
\hline Normal flow of pregnancy & $0,90 \pm 0,20$ & $1,70 \pm 0,20$ \\
\hline Arterial hypertension & $1,43 \pm 0,11$ & $2,48 \pm 0,17$ \\
\hline
\end{tabular}

Note: Differences are significant compared to the control

$*$ for $\mathrm{P}<0.01$ 


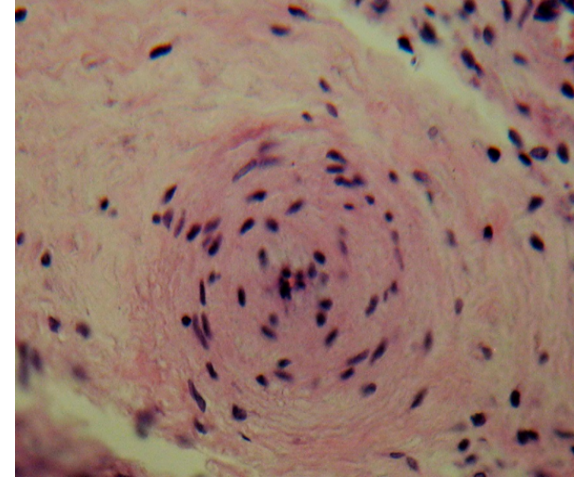

(A)

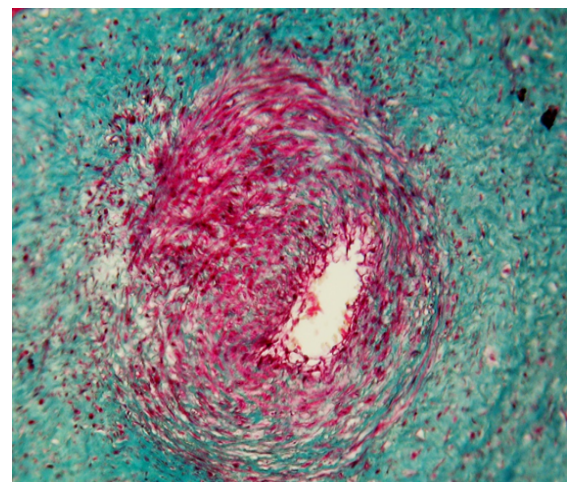

(C)

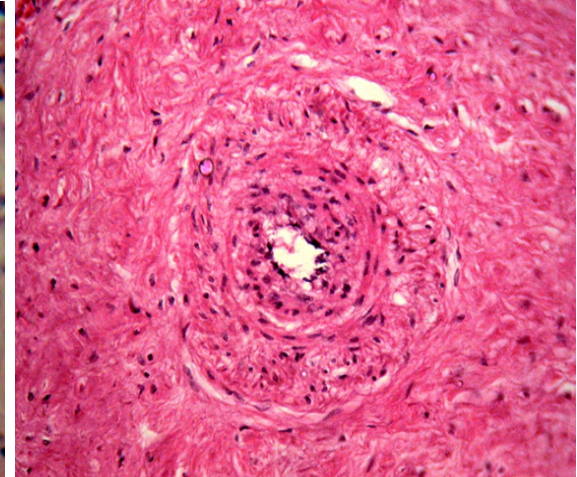

(B)

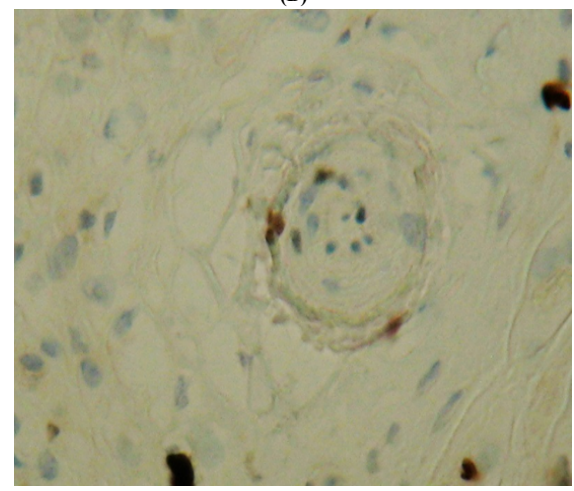

(D)

Figure 1. Arteries of the placenta chorion plate in hypertension conditions. (A,B) Staining with hematoxylin and eosin; (C) SHIK reaction with Masson color; (D) Immunohistochemical reaction to Ki-67 with Meyer's hematoxylin staining; $x$ 200. A - hypertrophy of tunica media and narrowing of the lumen of the artery; B - intertwining bundles of collagen fibers in the outer shell; C - high content of glycogen in smooth myocytes; D - expression of immunohistochemical marker Ki-67 in smooth myocytes.

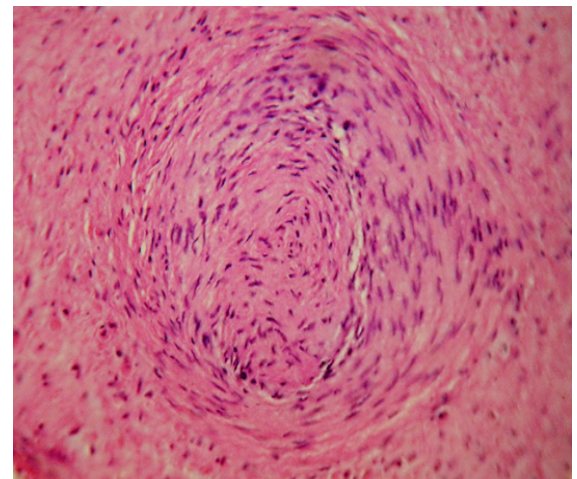

(A)

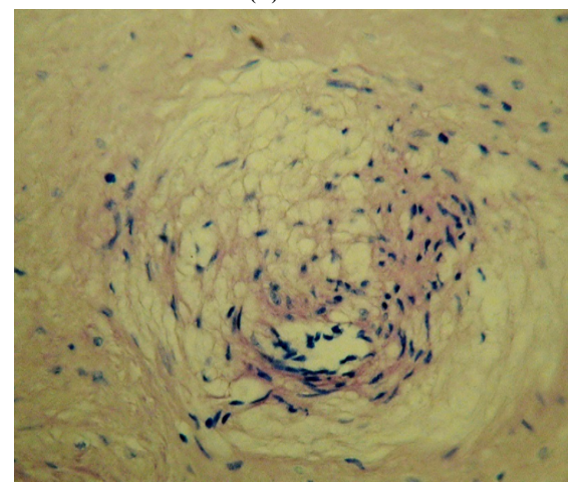

(C)

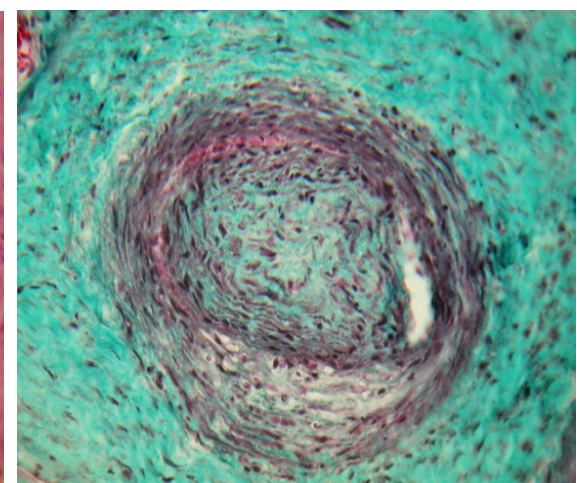

(B)

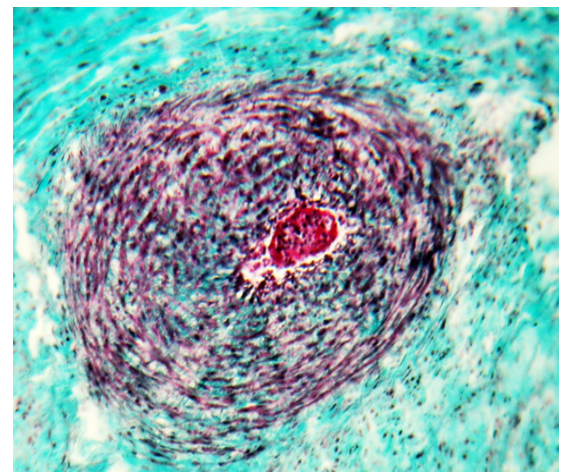

(D)

Figure 2. Pathological changes in the arteries of the chorionic plate of the placenta in conditions of arterial hypertension. Staining with hematoxylin and eosin (A); Masson (B, D) and van Gieson (C); x 200. A - a complex of smooth myocytes tunica intia, sharply narrowing the lumen of the artery; B - dystrophic changes of smooth inner and middle shell myocytes; C - edema of the artery wall; D - intravascular thrombus 
on the artery wall $[18,19]$. It is important to consider that the pressure (stress/strain) and blood flow (shift) can regulate the function [20], differentiation, proliferation and migration of vascular myocytes $[20,21]$. Proliferation of the main cellular populations of fetal arteries, registered in the course of this work, confirms the observations [22], indicating the vascularization of villi in the III trimester of pregnancy by the mechanisms of angiogenesis.

The study, conducted using the method of myocyte isolation, revealed significant differences in the composition of the population of the SM arteries of the HP of the placenta in areas with different hemodynamic conditions [9]. In branching and rotation of the vascular bed, muscle cells are distinguished by mitotic activity, an increase in the proportion of hypertrophied and polyploid forms. Earlier in our laboratory, it was shown that artery zones with flow dividers in the human fetus have additional regulatory smooth muscle complexes, usually in the inner shell [8], which are apparently formed due to migration of SM from tunica media $[23,24]$.

The population of vascular myocytes is not uniform [25]. The phenotype of small phase (fast) SM, capable of undergoing hypertrophic growth, and tonic (slow) vascular SMs undergoing hyperplasia has been identified. Load changes can cause phenotypic modulation of myocytes and proliferation of SM $[9,25]$. Thus, hemodynamic conditions may be a factor affecting the properties of the SM vascular wall. Phenotypic modulations of myocytes that occur in arteries characterized by high branching, anastomosing, many turns, are discussed for the initiation of the development of hypertension, heart failure, ischemia, obesity and diabetes [26].

Dystrophic changes in arteries in pregnant women with hypertension, found in this work, were previously described by a team of researchers from Brazil [27]. In groups with moderate and severe hypertension ( $>110 \mathrm{~mm} \mathrm{Hg}$ ), hyperplastic changes in the intima and middle layer and acute atherosis, manifested by fibrinoid necrosis, hyalinization of the vascular wall and infiltration of xanthomated histiocytes in the subintyme were noted.

Pathological changes noted in the study are manifested in a decrease in the capacity of the arterial channel HP. Identified structural changes lead to a significant decrease in blood volume, the development of acute or chronic placental anemia and, as a consequence, placental insufficiency and hypoxic conditions in the fetus.

\section{Conclusions}

In pregnant women with $\mathrm{AH}$, an increase in the pulsation index of the umbilical cord and a decrease in the minute volume of blood (cardiac output of the mother) indicate the development of fetal hypoxia.

Structural transformations of the arteries of the chorionic plate of the placenta in women with hypertension are manifested by an increase in the number of arteries with a functional layer of smooth myocytes in the middle shell, as well as vessels of the closing type with intimal muscles actively participating in the regulation of blood circulation.

Arterial hypertension can lead to a pronounced narrowing of the lumen of the arteries, and in some cases causes complete obliteration of the lumen. In arteries with a functional layer in tunica media, dystrophic changes of smooth myocytes, edema and intravascular thrombosis are detected.

\section{References}

1. Bryantsev MYu, Ochapkin MB, Trokhanova OV, Marchenko AI (2010) Prognostic value of the definition of central hemodynamics and portal blood flow in hypertension in the third trimester of pregnancy in relation to perinatal outcomes. Surgery 5: 55-61.
2. Glukhovets BI, Glukhovets NG, Tarasov VN (2002) Pathogenetic basis of hormonal endometrial hypertrophy in early spontaneous abortions. Arkh Patol 64: 28-31. [Crossref]

3. Guriev DL, Okhapkin MB, Karpov N.Yu, Blinov DV (2011) The course and outcome of pregnancy, complicated by preeclampsia, depending on the type of central maternal hemodynamics. Obstetrics and Gynecology 7: 14-19.

4. Milovanov AP (1999) Pathology of the mother-placenta-fetus system. Moscow Medicine.

5. Shabalov NP (2006) Neonatology. Moscow Medpress-information.

6. Kulida LV, Panova IA, Peretyatko LP (2008) Bulletin of gynecologists, obstetrics and perinatology $7: 25-28$.

7. Cocqueberi M, Bernodt S, Stgond N (2012) Comparative etxpression of hCG $\beta$-genes in human trophoblast from early and late first-trimester placentas. Am J Physiol Endocrinol Metab 303: 950-58.

8. Gansburgskiy AN, Yaltsev AV (2015) Features of the morphogenesis of the fetal blood vessels during placental insufficiency of pregnant women. Russian Herald of Perinatology and Pediatrics 60: 45-49.

9. Gansburgskiy AN, Yaltsev AV (2016) Morphology and ploidy of smooth myocytes of the chorion arteries under conditions of different hemodynamics. Bull Exper biol Med 162: $507-510$.

10. Caniggia I, Winter JL (2002) Adriana and Luisa Castellucci Award lecture 2001 Hypoxia inducible factor-1: oxygen regulation of trophoblast differentiation in normal and pre-eclamptic pregnancies-a review. Placenta 23: 47-57.

11. Lyall F, Myatt L (2002) The role of the placenta in pre-eclampsia--a workshop report. Placenta 23 Suppl A: S142-145. [Crossref]

12. Avtandilov GG (1990) Medical morphometry. Moscow Medicine.

13. Kaufman OYa (1987) Structural basis of adaptation and compensation of disturbed functions/ Ed. D.S. Sarkisov. Moscow Medicine: 131-53.

14. Intrauterine Human Development: A Guide for Physicians (2006) Ed. AP Milovanov, SV Saveliev. Moscow MDV.

15. Sarkisov DS, Paltsev MA, Khitrov NK (1997) General pathology of man. Moscow Medicine.

16. Kiladze AB, Dzhemukhadze NK (2013) Qualimetry in the histochemistry of enzymes Vologda Infra-Engineering.

17. Hovorka E (1970) The human placenta. Warsaw.

18. Gansburgskiy AN (1995) The structure of the arteries and features of hemodynamics in the mouth of outgoing vessels. Morphology 108: 82-91.

19. Karo K, Pedley T, Shroter R, Sid U (1981) Mechanics of blood circulation. Moscow Mir.

20. Fisher SA (2010) Vascular smooth muscle phenotypic diversity and function. Physiol Genomics 42A: 169-187. [Crossref]

21. Hao H, Gabbiani G, Bochaton-Piallat ML (2003) Arterial smooth muscle cell heterogeneity: implications for atherosclerosis and restenosis development. Arterioscler Thromb Vasc Biol 23: 1510-1520. [Crossref]

22. Milovanov AP, Erofeeva LM, Aleksandrovich NV, Zolotukhin IA (2012) Morphology of the human placenta in the second and third trimesters of pregnancy. Morphology 142: 64-67.

23. Jimenez M, Daret D, Choussat A, Bonnet J (1999) Immunohistological and ultrastructural analysis of the intimal thickening in coarctation of human aorta. Cardiovasc Res 41 : 737-745. [Crossref]

24. Hodebeck M, Scherer C, Wagner AH, Hecker M, Korff T (2014) TonEBP/NFAT5 regulates ACTBL2 expression in biomechanically activated vascular smooth muscle cells. Front Physiol 5: 467-68. [Crossref]

25. Shekhonin BV, Tararak EM, Griaznov OG, Zotikov AE, Pokrovskiī AV (2003) Phenotypes of smooth muscle cells in carotid arteries in Takayasu's disease. Arkh Patol 65: 31-35. [Crossref]

26. Owens GK, Kumar MS, Wamhoff BR (2004) Molecular regulation of vascular smooth muscle cell differentiation in development and disease. Physiol Rev 84: 767-801. [Crossref]

27. de Sousa FLP, Sass N, Camano L, Stávale J N, de Sousa Mesquita MR, et al. (2008) Estudo da morfologia vascular do leito placentário na hipertensão arterial crônica. Rev Assoc Med Bras São Paulo 54: 483-485.

Copyright: (C2018 Gansburgskiy AN. This is an open-access article distributed under the terms of the Creative Commons Attribution License, which permits unrestricted use, distribution, and reproduction in any medium, provided the original author and source are credited. 